\title{
Long-Term Suppression of Viral Replication in Chronic Hepatitis B: Outcomes and Future Directions
}

\author{
Jae-Jun Shim \\ Department of Internal Medicine, Kyung Hee University School of Medicine, Seoul, Korea
}

See "Disease Progression in Chronic Hepatitis B Patients under Long-Term Antiviral Therapy" by Jin Chang Moon, et al. on page 395, Vol. 9. No. 3, 2015

When considering the cost-effectiveness of a new treatment, our usual approach is to find the optimal treatment candidates, rather than a way to lower the cost. This is because the longterm efficacy of the new treatment is unclear initially, and as clinicians we are more interested in the patients than in the price. In this situation, to show the most dramatic and beneficial effects, we define the optimal candidates as those patients who are at a sufficiently high risk. This approach has been applied in many clinical trials, treatment guidelines, and insurance reimbursement policies.

In chronic hepatitis $\mathrm{B}(\mathrm{CHB})$, the most at-risk patients have been defined based on long-term observational studies. ${ }^{1}$ The higher-risk group has been defined using arbitrary serum alanine aminotransferase (ALT; 1- to 2-fold the upper normal limit) and hepatitis B viral (HBV) DNA levels (2,000 or 20,000 IU/mL) and liver biopsy findings, if available.

Note, however, that all patients with chronic HBV infection are at risk of disease progression. Even patients who are inactive HBV carriers are at 4.6-fold greater risk of developing hepatocellular carcinoma (HCC) than noninfected subjects. ${ }^{2}$ Therefore, complete eradication of HBV should be the ideal treatment goal. Since it is very difficult to eradicate covalently closed circular DNA (cccDNA) in hepatocytes, the current practical goal is to maintain suppression of viral replication. ${ }^{3}$ To prevent disease progression to cirrhosis, end-stage liver disease, and HCC, most patients with proven active HBV replication should be considered as candidates for antiviral therapy.

In practice, only a minority of these patients receive antiviral therapy. This might be due to the low recognition rate of HBV infection, but also to unmet treatment indications. In the clinical setting, many patients defer antiviral therapy because of relatively low serum ALT or HBV DNA levels. Their viral states are between those of stable (inactive HBV carriers) and high-risk patients (candidates for antiviral therapy). In one study, of the patients with chronic HBV infection, 44\% had serum HBV DNA levels exceeding 2,000 IU/mL. Of these, however, only 9\% had a serum ALT level exceeding $45 \mathrm{IU} / \mathrm{L}$ at baseline. During a followup of 13 years, 51\% never had elevated ALT levels (>45 IU/L), ${ }^{4}$ albeit active HBV replication (>2,000 IU/mL). This suggests that half of these patients were having difficulty taking their antiviral therapy. If we were to use the more stringent indication from the Korean National Health Insurance guidelines (aspartate aminotransferase or ALT >80 IU/L and HBV DNA >2,000 to 20,000 $\mathrm{IU} / \mathrm{mL}$ ), there is no doubt that many more patients would be rejected as treatment candidates. Nevertheless, although the risk faced by this group is much lower than that of the high-risk group, these patient numbers are extremely large, accounting for one-third of all HCC cases. ${ }^{4}$

Currently, long-term outcome results of patients with $\mathrm{CHB}$ treated with antiviral therapy are accumulating. ${ }^{5-7}$ The results are promising. Hepatic fibrosis and cirrhosis tend to regress in the majority of patients. ${ }^{5}$ Nevertheless, these studies clearly show that a minority of patients experience disease progression despite long-term antiviral therapy. Although the risk for HCC development is reduced markedly, it is still higher than that of inactive carriers or normal subjects, necessitating cautious surveillance. $^{7-9}$ This suggests that the current outcomes of antiviral therapy are imperfect, and thus further effort is needed to improve patient quality of life and survival.

In this journal, Moon et al. ${ }^{10}$ followed 524 patients with $\mathrm{CHB}$ on nucleoside analog therapy for a median of 48 months. Of these, 194 had liver cirrhosis at baseline. Most patients were

Correspondence to: Jae-Jun Shim

Department of Internal Medicine, Kyung Hee University Hospital, 23 Kyungheedae-ro, Dongdaemun-gu, Seoul 130-872, Korea

Tel: +82-2-958-8155, Fax: +82-2-968-1848, E-mail: joyshim@khu.ac.kr pISSN 1976-2283 eISSN 2005-1212 http://dx.doi.org/10.5009/gnl15105

@ This is an Open Access article distributed under the terms of the Creative Commons Attribution Non-Commercial License (http://creativecommons.org/licenses/by-nc/3.0) which permits unrestricted non-commercial use, distribution, and reproduction in any medium, provided the original work is properly cited. 
high risk or at an advanced stage. The authors investigated the disease progression rate and factors associated with progression and found that 67 patients (12.8\%) experienced disease progression, including progression to cirrhosis (21 patients), development of cirrhosis-related complications (46 patients), HCC (34 patients), and liver-related death (five patients). Disease progression was associated with age $>50$ years (hazard ratio [HR], 1.05) and cirrhosis (HR, 2.95). HCC development was associated with age $>50$ years, family history, and cirrhosis. A maintained virological response for more than 12 months was associated with a lower risk of disease progression.

The results are consistent with recent reports, thus demonstrating the current picture of antiviral therapy and the limitations to be overcome. The authors showed that if patients are older than 50 years or cirrhotic at the time of drug initiation, the disease can progress despite long-term antiviral therapy. Nevertheless, this does not imply that all patients with $\mathrm{CHB}$ should start antiviral therapy before 50 years of age or before progression to liver cirrhosis. This could be confirmed by large randomized studies, which, unfortunately, are difficult to conduct due to the need to evaluate a large number of subjects over a very long follow-up period.

The study questioned the current recommendations for antiviral therapy and the wait-and-monitor strategy. Although it is difficult to draw conclusions using the current data, we should ask ourselves, "Is it really acceptable to wait until patients progress to higher risk stages before satisfying the current treatment indications?"

The study showed the beneficial effects of antiviral therapy, while suggesting that more effort is needed to identify unrecognized patients before it is too late. Another critical issue for patients on antiviral therapy is maintaining sustained viral suppression. We should choose a potent agent with an optimal resistance profile as first-line therapy and monitor HBV DNA levels and patient compliance regularly. In addition, to increase the cost-effectiveness of antiviral therapy, we should find a more systematic approach to reduce the price.

\section{CONFLICTS OF INTEREST}

No potential conflict of interest relevant to this article was reported.

\section{REFERENCES}

1. Chen CJ, Yang HI, Su J, et al. Risk of hepatocellular carcinoma across a biological gradient of serum hepatitis B virus DNA level. JAMA 2006;295:65-73.

2. Chen JD, Yang HI, Iloeje UH, et al. Carriers of inactive hepatitis B virus are still at risk for hepatocellular carcinoma and liver-related death. Gastroenterology 2010;138:1747-1754.

3. Suk KT, Baik SK, Yoon JH, et al. Revision and update on clinical practice guideline for liver cirrhosis. Korean J Hepatol 2012;18:1-21.

4. Chen CF, Lee WC, Yang HI, et al. Changes in serum levels of HBV DNA and alanine aminotransferase determine risk for hepatocellular carcinoma. Gastroenterology 2011;141:1240-1248.e2.

5. Marcellin P, Gane E, Buti M, et al. Regression of cirrhosis during treatment with tenofovir disoproxil fumarate for chronic hepatitis B: a 5-year open-label follow-up study. Lancet 2013;381:468-475.

6. Niro GA, Ippolito AM, Fontana R, et al. Long-term outcome of hepatitis B virus-related chronic hepatitis under protracted nucleos(t)ide analogues. J Viral Hepat 2013;20:502-509.

7. Park YH, Kim BK, Kim JK, et al. Long-term outcomes of chronic hepatitis B virus infection in the era of antiviral therapy in Korea. J Gastroenterol Hepatol 2014;29:1005-1011.

8. Cho JY, Paik YH, Sohn W, et al. Patients with chronic hepatitis $\mathrm{B}$ treated with oral antiviral therapy retain a higher risk for HCC compared with patients with inactive stage disease. Gut 2014;63: 1943-1950.

9. Idilman R, Gunsar F, Koruk M, et al. Long-term entecavir or tenofovir disoproxil fumarate therapy in treatment-naive chronic hepatitis B patients in the real-world setting. J Viral Hepat 2015;22:504-510.

10. Moon JC, Kim SH, Kim IH, et al. Disease progression in chronic hepatitis B patients under long-term antiviral therapy. Gut Liver 2015;9:395-404. 